\title{
Nanoscopic visualization of soft matter using fluorescent diarylethene photoswitches
}

\author{
Oleksii Nevskyi, ${ }^{[a]}$ Dmytro Sysoiev,${ }^{[b]}$ Alex Oppermann, ${ }^{[a]}$ Thomas Huhn, ${ }^{[b]}$ and Dominik Wöll ${ }^{*[a]}$
}

\begin{abstract}
The in situ imaging of soft matter is of paramount importance for a detailed understanding of functionality on the nanoscopic scale. Even though superresolution fluorescence microscopy methods with their unprecedented imaging capabilities have revolutionized research in life sciences, this potential has so far only little been exploited in materials science. One of the main obstacles for a more universal application of superresolved fluorescence microscopy methods is the limitation of readily available suitable dyes with the sophisticated photophysical and photochemical properties required to overcome the diffraction limit. Here, we report a novel diarylethene-based photoswitch with a highly fluorescent closed and a non-fluorescent open form. Its photophysical properties, its switching behavior and its high photostability make the dye most ideal for photoactivation localization microscopy (PALM). We demonstrate its capability to resolve apolar structures without the need of any additives with an accuracy far below the diffraction limit of optical light in cylindrical micelles formed by amphiphilic block copolymers.
\end{abstract}

The nanoscopic structure determines the properties of soft matter materials. ${ }^{[1]}$ Therefore, methods to directly visualize structures in the nanometer range are of paramount importance for the ongoing evolution of novel materials with specialized and adaptive properties for sophisticated applications. Scanning probe microscopy techniques give access to the nanometer range and determine surface properties such as topology and softness. $^{[2,3]}$ Modern electron microscopy methods such as scanning electron microscopy $(\mathrm{SEM})^{[4,}$ 5] and transmission electron microscopy $\left(\right.$ TEM) ${ }^{[6-8]}$ can yield structural information even in the subnanometer range for sufficient electron density contrast. Despite the success of these methods, they are technically demanding and time-consuming. Furthermore, many soft matter samples possess poor electron contrast, and require non-invasive in situ imaging beyond the surface with the possibility to directly study dynamics. In recent years, superresolved fluorescence microscopy has revolutionized optical imaging, ${ }^{[9-14]}$ utilizing photophysical or photochemical switching of fluorescent dyes in a sophisticated manner in combination with modern optics. So far, the life sciences in particular have benefitted from the new possibilities of resolving structures well beyond the diffraction limit of light. Only few examples for superresolution microscopy in material science have been reported ${ }^{[15-18]}$ since concepts that require for example the addition of (polar) switching buffers often fail for these systems. Therefore, the main bottleneck for more universal

[a] O. Nevskyi, A. Oppermann, Prof. Dr. D. Wöll Institute for Physical Chemistry

RWTH Aachen University

Landoltweg 2, 52074 Aachen

E-mail: woell@pc.rwth-aachen.de

[b] Dr. D. Sysoiev, Dr. T. Huhn

Department of Chemistry

University of Konstanz

Universitätsstr. 10, 78464 Konstanz

Supporting information for this article is given via a link at the end of the document. applications of superresolution imaging are switchable dyes with suitable (photo-)physical and chemical properties such as high photostability, adjustable switching rates, minimum interaction with the environment to be probed and simple design, with the possibility of multiple and straight-forward derivatization for the specific labelling of structures or compartments. ${ }^{[19]}$

Diarylethenes represent a powerful class of organic photoswitches with various applications such as data storage and chemical sensing. ${ }^{[20-22]}$ Despite their wide variability, the lack of derivatives with strong fluorescence in the visible range, i.e. of the closed form, has prevented their direct application for superresolution imaging. Strategies to covalently link diarylethene switches with suitable fluorophores and to utilize energy $^{[23]}$ or electron transfer ${ }^{[24]}$ of the resulting multichromophoric systems have been successful, but require extended synthesis, and result in rather large probes. Labelling structures with such extended probes can significantly alter the structures to be visualized. ${ }^{[23,25]}$ As an alternative, suitable modification of the diarylethene photoswitch itself could directly give access to the envisioned properties. Uno et al. ${ }^{[26,27]}$ found that photochromic diarylethenes derivatives bearing sulfon groups can fluoresce in the closed form which inspired our research.

Here, we present a novel photochromic diarylethene photoswitch for superresolution microscopy bearing high photostability and strong fluorescence in its closed form. We investigate its photophysical and photochemical properties in solution and in polymers. The outstanding performance for photoactivated localization microscopy (PALM) of soft matter structures is demonstrated by imaging the self-assembly of block copolymers. In dense surrounding, the switching behavior can be used to probe free volume on a molecular basis.

The photoswitch (see Figure 1a) was synthesized by a Suzuki-Miyaura coupling reaction using the 6,6'-diiodo derivative of 1,2-bis(2-ethyl-1,1-dioxidobenzothiophene-3-yl)perfluorocyclopentene and naphthalene-2-ylboronic acid as described in the Supporting Information. In the following, we will demonstrate that the photoswitch meets all requirements of an ideal dye for superresolved fluorescence microscopy: ${ }^{[28,29]}$ spectrally wellseparated on- and off-states, high absorption coefficients, high fluorescence quantum yield of the on-state, high photostability, a low on $\rightarrow$ off-switching quantum yield, and a reasonable off $\rightarrow$ onswitching quantum yield with the possibility of tuning the off $\rightarrow$ onswitching rate by the intensity of the activation laser. As will be shown below, especially the lower off-switching quantum yield of the naphthyl compared to the phenyl derivative ${ }^{[26]}$ is of significant advantage for superresolved localization microscopy.

The structure of our diarylethene photoswitch and its absorption spectra in the open and closed form are presented in Figure 1. The open form does not absorb in the visible region and therefore appears colorless. In contrast, the absorption of the closed form ranges up to $550 \mathrm{~nm}$ with a maximum at 475 $\mathrm{nm}$. In apolar media, the closed form exhibits strong fluorescence with a fluorescence quantum yield of 0.65 (as determined in solution in 1,4-dioxane) in the wavelength range between 500 and $650 \mathrm{~nm}$ (see Figure $1 \mathrm{~b}$ ). In more polar solvents, the quantum yield drops significantly and a bathochromic shift of the fluorescence of the closed form is observed (see Table S2 in the Supporting Information). The wavelength range of fluorescence can be tuned by proper substitution. 
WILEY-VCH

The open and closed form can be converted into each other by irradiation with suitable wavelengths (see Figure 2). Irradiation in the UV mainly results in formation of the closed form, whereas wavelengths between 400 and $550 \mathrm{~nm}$ recover the open form. After sufficient irradiation, a photostationary equilibrium is reached which depends on the absorption coefficient of both species at the corresponding wavelength and their conversion quantum yields. For superresolution microscopy, a photoswitch should emit thousands of photons before switching back to its dark form, thus enabling the collection of a sufficient amount of photons to enable good localization accuracy. ${ }^{[30]}$ Therefore, the on-off switching quantum yield should be clearly below 0.0001 . On the other hand, the rather efficient on-switching of the dark state is of advantage since only small UV activation intensity is required. Our novel photoswitch excellently fulfills both of these requirements as discussed below.

The quantum yield $\phi_{0 \rightarrow c}$ of the cyclization was determined to be 0.045 in 1,4-dioxane (for calculation see the Supporting Information). Thus, the fluorescent (closed) form of the photoswitch can be easily populated with low UV light intensity. Correspondingly, using a high power $488 \mathrm{~nm}$ LED, we determined a quantum yield $\phi_{\mathrm{c} \rightarrow 0}$ of $6.9 \times 10^{-6}$ for the cycloreversion. The extremely low quantum yield for the off-switching process is of most essential advantage for PALM imaging since it ascertains enough photons to be collected for high accuracy localization of the position of single photoswitches before turning dark. Similar quantum yields are also obtained on the single molecule level for photoswitches embedded in thin polymer films (vide infra). In these measurements, we could additionally certify that the disappearance of molecules was mainly due to switching and not due to an irreversible photo damage because in most cases the fluorescence of molecules could be reactivated with UV light. In the case of the photoswitch in PS, on average $1.96 \times 10^{3}$ photons per cycle could be collected (see the Supporting Information). From this, the possible localization accuracy was estimated according to Mortensen et al. ${ }^{[30]}$ to be $20 \mathrm{~nm}$. More details for the evaluation of the photokinetics can be found in the Supporting Information.

a)

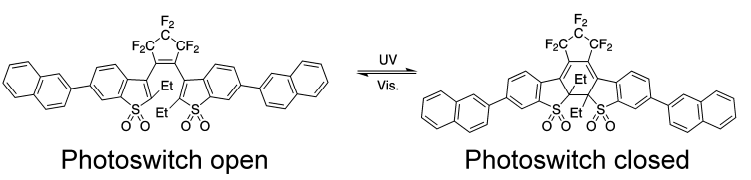

b)
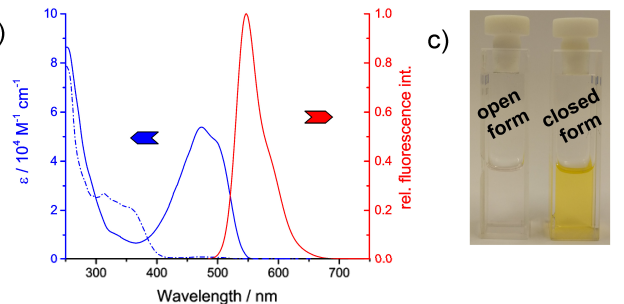

d)

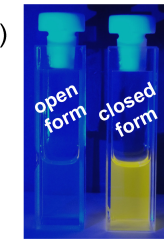

Figure 1. a) Chemical structures of the open and the closed form of the photoswitch; b) absorption spectra of the open (blue dashed line) and closed form (blue solid line) and fluorescence spectrum of the closed form (red); c) photos of the open (left) and closed (right) form of the photoswitch dissolved in 1,4-dioxane; d) photos open (left) and closed (right) form of the photoswitch dissolved in 1,4-dioxane upon $488 \mathrm{~nm}$ excitation.
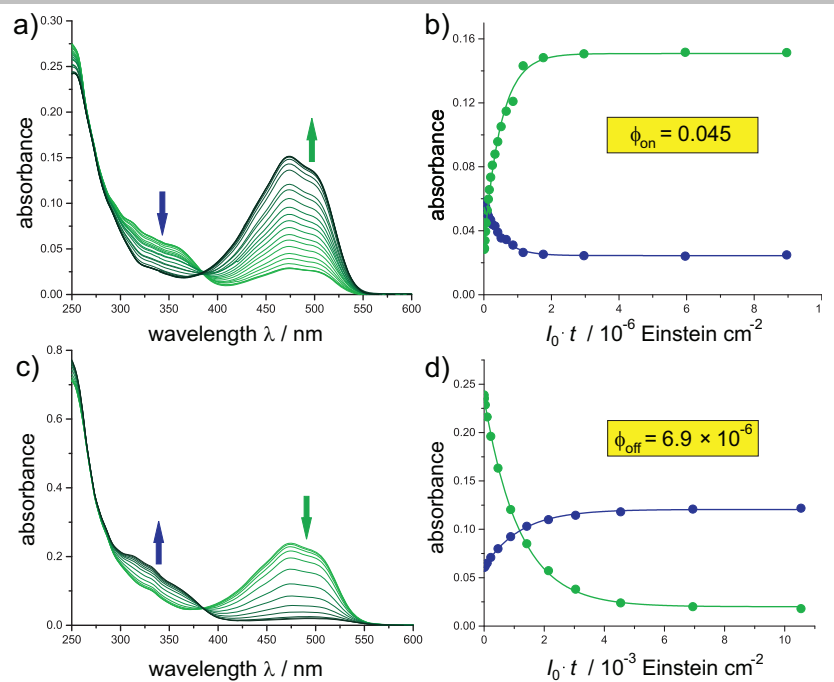

Figure 2. Evolution of the absorption spectra (measured in an $1 \mathrm{~cm}$ cuvette) and kinetics of the photoswitch in 1,4-dioxane during on- and off-switching, respectively: a) on-switching from the open to the closed form under UV light irradiation $(366 \mathrm{~nm})$ until reaching the photostationary state (almost exclusively the closed form); b) time-evolution of the absorbance at $475 \mathrm{~nm}$ (green circles) and at $342 \mathrm{~nm}$ (blue circles) upon irradiation with $366 \mathrm{~nm}$. c) off-switching from the closed to the open form under irradiation with $488 \mathrm{~nm}$ until reaching the photostationary state (almost exclusively the open form). d) time-evolution of the absorbance at $475 \mathrm{~nm}$ (green circles) and at $342 \mathrm{~nm}$ (blue circles) upon irradiation with $488 \mathrm{~nm}$; the data in (b) and (d) were fitted according to monoexponential kinetics and the resulting switching quantum yields are shown. The abscissa is presented as light dose in Einstein, i.e. mol photons, per square centimetres. The calculation of the quantum yields $\phi_{\text {on }}$ and $\phi_{\text {off }}$ is described in the Supporting Information.

The switching kinetics of the photoswitch depends on its environment. This dependency is especially pronounced in dense systems of rather high viscosity. Therefore, in such environments, choosing appropriate conditions such as timing and intensity of the illumination is important to achieve good superresolved images. On the other hand, this dependency can be used to obtain environmental information in addition to structure. We investigated the switching behavior of the photoswitch embedded in spin-coated polymer films of poly(ethylacrylate) (PEA), poly(n-butylmethacrylate) (PBMA), poly(styrene) (PS), and poly(methylmethacrylate) (PMMA). Representatively, the switching behavior in a PBMA film is shown in Figure 3 (for the other polymers see the Supporting Information). We counted the number of fluorescent molecules within the $40 \times 40 \mu^{2}$ area of widefield microscopy images during laser illumination with $488 \mathrm{~nm}$ (ca. $0.20 \mathrm{~kW} \mathrm{~cm}$ ) and each $5 \mathrm{~s}$ activated molecules with a laser at $380 \mathrm{~nm}$ (illumination duration $1 \mathrm{~s}$, intensity ca. $0.62 \mathrm{~kW} \mathrm{~cm}^{-2}$ ). The switching kinetics can be accessed by evaluation of the temporal evolution of the amount of on-molecules within activation cycles (see Figure 3 ). The number of fluorescent molecules was determined to decrease with first order kinetics with a rate constant of $1.02 \mathrm{~s}^{-1}$ corresponding to an off-switching quantum yield $\phi_{\mathrm{c} \rightarrow 0}$ of $2.7 \times 10^{-5}$ (for the other polymers see Figure S4 in the Supporting Information). We found that the off-switching rate depends on the glass transition temperature $\left(T_{\mathrm{g}}\right)$ of the embedding polymer and is higher for polymers with lower $T_{\mathrm{g}}$. The quantum yield varies from $5.3 \times 10^{-6}$ in PS to $2.7 \times 10^{-5}$ in PEA. Additional to the different kinetics, a significant fraction of molecules did not switch off at all in the higher $T_{\mathrm{g}}$ polymers (see the Supporting Information). This points towards a heterogeneous distribution of free volume as previously discussed using single molecule rotation measurements, ${ }^{[31]}$ and, for polymers with different $T_{\mathrm{g}}$ 's, using single molecule photoinduced electron transfer studies. ${ }^{[32,33]}$

Besides photoswitching kinetics, the photostability of probes determines their suitability for superresolution fluorescence microscopy techniques. As shown in Figure 3, our 
photoswitch can perform many on-off cycles before photobleaching. This might be of minor importance for probing a fixed structure where one localization event per fluorophore followed by irreversible photodamage is sufficient. For probing dynamics, however, a quickly decreasing amount of activatable switches is not desirable. Here, photoswitches with high cycle numbers are of enormous advantage. We could switch most of our photoswitches more than 100 times before irreversible photodamage occurred.

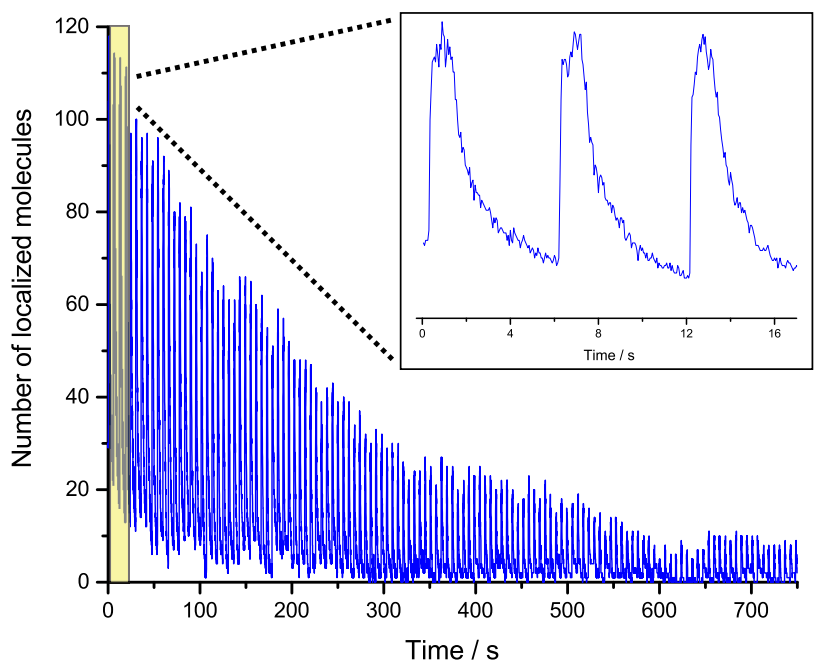

Figure 3. Switching cycles of the photoswitch embedded into a thin PBMA film upon $488 \mathrm{~nm}\left(0.20 \mathrm{~kW} / \mathrm{cm}^{2}\right)$ excitation and UV-activation each $5 \mathrm{~s}$. The inset shows a zoom into the first $17 \mathrm{~s}$ where the exponential off-switching can be recognized.

In the following, we will demonstrate the capability of our photoswitch for visualizing apolar structures with a resolution well below the diffraction limit of light. Amphiphilic polystyrenepolyethene oxide block copolymers (PS- $b$-PEO, $M_{\mathrm{n}, \mathrm{PS}}=38 \mathrm{~kg}$ $\mathrm{mol}^{-1}, M_{\mathrm{n}, \mathrm{PEO}}=11 \mathrm{~kg} \mathrm{~mol}^{-1}$ ) were self-assembled with a small amount of our photoswitch added. Using appropriate conditions, the morphology of the formed nanostructures can be well controlled. ${ }^{[34,35]}$ As described in the Supporting Information, we chose conditions to yield cylindrical micelles with a hydrophobic PS core labelled with our diarylethene photoswitches and a hydrophilic PEO block shell. After drying the formed cylindrical structures, fluorescence microscopy movies were recorded under appropriate laser intensities to allow for well-suited single photoswitch blinking kinetics (for details see the Supporting Information). As already mentioned above, some dyes do not switch in PS. They were photobleached with the $488 \mathrm{~nm}$ laser prior to the measurements. Analysis with ThunderSTORM ${ }^{[36]}$ yielded superresolved PALM images as representatively shown in Figure 4a. On the right superresolved part of the image, structural details can be detected which are obscure in the diffraction-limited left part of the image. In Figure $4 \mathrm{~b}$, we show a representative superresolved $\mathrm{Y}$-shaped structure and demonstrate the capability of resolving structures well below the diffraction limit. Intensity profiles along different vertical lines reveal that we can easily resolve cylinders with separation of 84 $\mathrm{nm}$. It should be noted that the resolution here is not limited by the properties of the dyes but by the width of the labelled cores. These exhibit a core diameter of ca. $50 \mathrm{~nm}$ as estimated from the width of the cylinders of $62 \mathrm{~nm}$ as determined by AFM (see Figure $\mathrm{S} 6$ in the Supporting Information). Taking into account that, as shown in Figure $4 \mathrm{~b}$, the cylinders were imaged with a full-width at half-maximum (FWHM) of ca. $70 \mathrm{~nm}$ and that this value is obtained by a convolution of the real size of the labelled core with the accuracy of single molecule localization, we can estimate the resolution to be approx. $50 \mathrm{~nm}$ related to FWHM or $21 \mathrm{~nm}$ concerning standard deviation. This means that our photoswitch allows for PALM with a 10-fold increase of resolution with respect to the diffraction-limit.
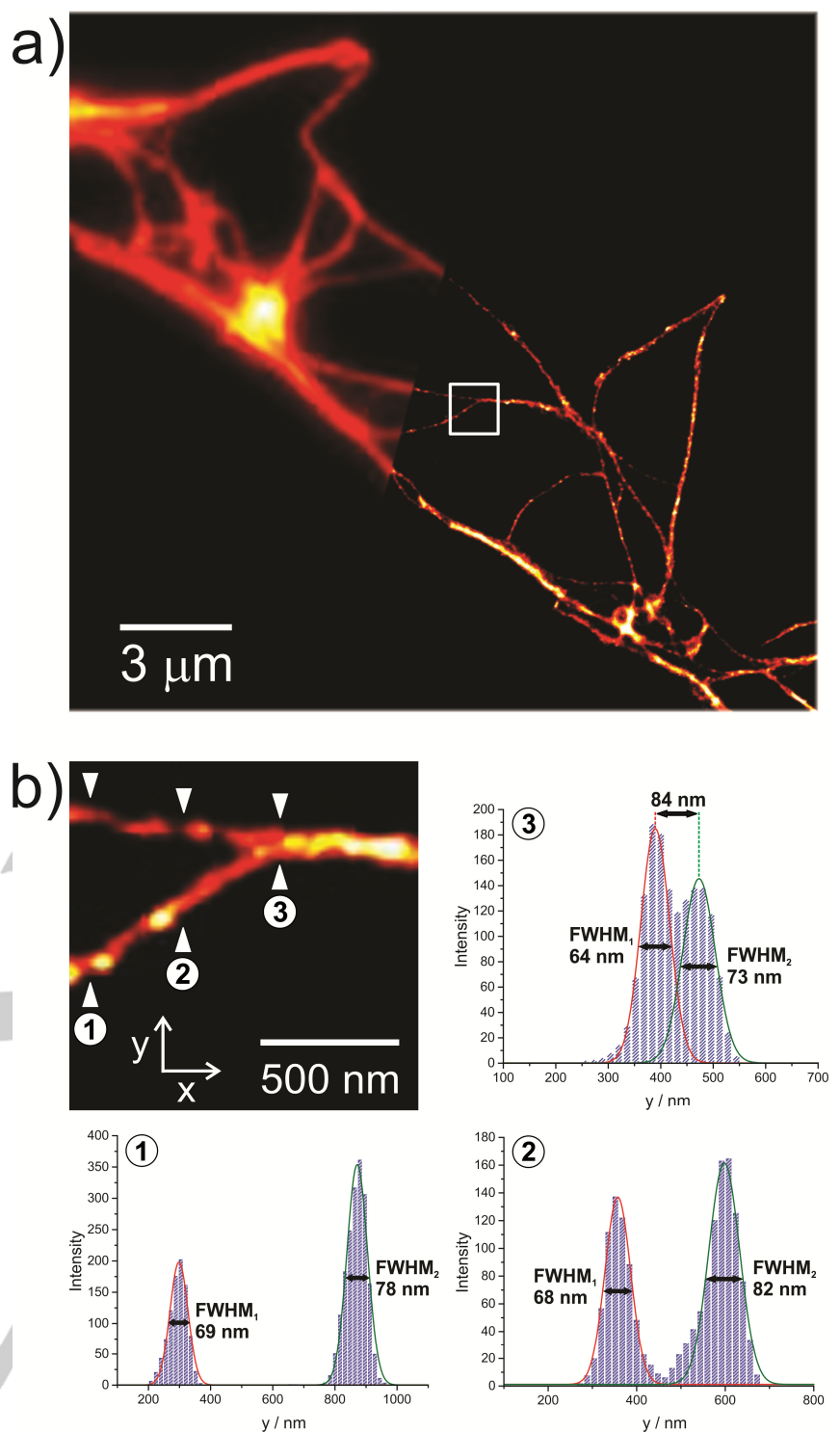

Figure 4. a) Conventional (left part) and superresolved (right part) image of block copolymer cylindrical micelles forming string-structures recorded using PALM; b) resolution of the Y-shaped structure in PALM images: at three different $\mathrm{x}$-positions, intensity profiles along the vertical $\mathrm{y}$-axis are presented.

In conclusion, we utilized for the first time a fluorescent photochromic diarylethene derivative for superresolved photoactivation localization microscopy. We rationalized its suitability for superresolved imaging by an evaluation of the photoswitching kinetics and found a sensitivity of photoswitching which depends on the surrounding and which can be used as an independent readout to learn about local polymer density. We visualized the apolar core of selfassembled amphiphilic blockcopolymer nanostructures with a resolution far beyond the diffraction limit of light using PALM imaging. The applicability of the photoswitch is, however, not restricted to PALM but can be also used for reversible saturable optical fluorescence transitions (RESOLFT) microscopy ${ }^{[37]}$ and super-resolution optical fluctuation imaging (SOFI). ${ }^{[38]}$ The synthesis of these powerful photoswitches is straight-forward and a variety of substitutions using C-C coupling strategies is currently under way to specifically adapt their properties to the systems to be visualized. With suitable substitutions or embedding the chromophore into appropriate nanoparticles, we also envision the possibility of expanding the 
applicability of the probe to imaging in polar media. In summary, we believe that our results lay the foundation for applying diarylethene derivatives for superresolution microscopy of systems which could not be accessed so far due to the limitation in available dyes with suitable properties.

\section{Experimental Section}

The experimental details can be found in the Supporting Information.

\section{Acknowledgements}

We thank Patrick Wünnemann for valuable discussions on atomic force microscopy measurements and Stefan Walta for his assistance in fluorescence lifetimes measurements. Furthermore, we thank the German Research Foundation (DFG) for support within the SFB 985 "Functional Microgels and Microgel Systems".

Keywords: photoswitch • superresolution • fluorescence microscopy $\bullet$ photochemistry $\bullet$ blockcopolymers

[1] K. Matyjaszewski, Science 2011, 333, 1104-1105

[2] P. J. M. Stals, Y. Li, J. Burdyńska, R. Nicolaÿ, A. Nese, A R. A. Palmans, E. W. Meijer, K. Matyjaszewski, S. S. Sheiko, J. Am. Chem. Soc. 2013, 135, 11421-11424.

[3] E. T. Herruzo, A. P. Perrino, R. Garcia, Nat. Commun 2014, 5, 3126

[4] K. Geisel, L. Isa, W. Richtering, Angew. Chem. Int. Ed. 2014, 53, 4905-4909.

[5] C. Stoffelen, J. Voskuhl, P. Jonkheijm, J. Huskens, Angew. Chem. Int. Ed. 2014, 53, 3400-3404.

[6] H. Cui, Z. Chen, S. Zhong, K. L. Wooley, D. J. Pochan, Science 2007, 317, 647-650.

[7] R. Fenyves, M. Schmutz, I. J. Horner, F. V. Bright, J. Rzayev, J. Am. Chem. Soc. 2014, 136, 7762-7770

[8] C. V. Synatschke, T. Nomoto, H. Cabral, M. Förtsch, K. Toh, Y. Matsumoto, K. Miyazaki, A. Hanisch, F. H. Schacher, A. Kishimura, N. Nishiyama, A. H. E. Müller, K. Kataoka, ACS

Nano 2014, 8, 1161-1172.

[9] E. Betzig, G. H. Patterson, R. Sougrat, O. W. Lindwasser S. Olenych, J. S. Bonifacino, M. W. Davidson, J. LippincottSchwartz, H. F. Hess, Science 2006, 313, 1642-1645.

[10] M. Heilemann, S. van de Linde, M. Schüttpelz, R. Kasper, B. Seefeldt, A. Mukherjee, P. Tinnefeld, M. Sauer, Angew. Chem. Int. Ed. 2008, 47, 6172-6176.

[11] M. J. Rust, M. Bates, X. Zhuang, Nat. Methods 2006, 3 793-796.

[12] S. W. Hell, Science 2007, 316, 1153-1158.

[13] J. B. Grimm, T. Klein, B. G. Kopek, G. Shtengel, H. F. Hess, M. Sauer, L. D. Lavis, Angew. Chem. Int. Ed. 2016, 55, 1723-1727.

[14] W. R. Legant, L. Shao, J. B. Grimm, T. A. Brown, D. E. Milkie, B. B. Avants, L. D. Lavis, E. Betzig, Nat. Methods 2016, 13, 359-365.

[15] M. W. Gramlich, J. Bae, R. C. Hayward, J. L. Ross, Opt. Express 2014, 22, 8438-8450

[16] Z. Tian, A. D. Q. Li, D. Hu, Chem. Commun. 2011, 47, 1258-1260.

[17] C. K. Ullal, S. Primpke, R. Schmidt, U. Böhm, A. Egner, P. Vana, S. W. Hell, Macromolecules 2011, 44, 7508-7510.

[18] A. Aloi, A. Vargas Jentzsch, N. Vilanova, L. Albertazzi, E. W. Meijer, I. K. Voets, J. Am. Chem. Soc. 2016, 138, 2953-2956. [19] G. T. Dempsey, J. C. Vaughan, K. H. Chen, M. Bates, X. Zhuang, Nat. Methods 2011, 8, 1027-1036.
[20] M. Irie, T. Fukaminato, K. Matsuda, S. Kobatake, Chem. Rev. 2014, 114, 12174-12277.

[21] S. Fredrich, R. Göstl, M. Herder, L. Grubert, S. Hecht, Angew. Chem. Int. Ed. 2016, 55, 1208-1212.

[22] D. Kim, J. E. Kwon, S. Y. Park, Adv. Opt. Mat. 2016, 4, 790-797.

[23] C. Li, H. Yan, L.-X. Zhao, G.-F. Zhang, Z. Hu, Z.-L. Huang, M.-Q. Zhu, Nat. Commun. 2014, 5, 5709.

[24] T. Fukaminato, T. Doi, N. Tamaoki, K. Okuno, Y. Ishibashi, H. Miyasaka, M. Irie, J. Am. Chem. Soc. 2011, 133, 4984-4990.

[25] C. Li, Z. Hu, M. P. Aldred, L. X. Zhao, H. Yan, G. F. Zhang, Z. L. Huang, A. D. Q. Li, M. Q. Zhu, Macromolecules 2014, 47, 8594-8601.

[26] K. Uno, H. Niikura, M. Morimoto, Y. Ishibashi, H. Miyasaka, M. Irie, J. Am. Chem. Soc. 2011, 133, 13558-13564.

[27] F. Gillanders, L. Giordano, S. A. Diaz, T. M. Jovin, E. A. Jares-Erijman, Photochem. Photobiol. Sci. 2014, 13, 603-612. [28] A. Fürstenberg, M. Heilemann, Phys. Chem. Chem. Phys. 2013, 15, 14919-14930.

[29] M. Heilemann, P. Dedecker, J. Hofkens, M. Sauer, Laser Photonics Rev. 2009, 3, 180-202.

[30] K. I. Mortensen, L. S. Churchman, J. A. Spudich, H. Flyvbjerg, Nat. Methods 2010, 7, 377-381.

[31] A. Deres, G. A. Floudas, K. Müllen, M. Van der Auweraer, F. De Schryver, J. Enderlein, H. Uji-i, J. Hofkens,

Macromolecules 2011, 44, 9703-9709.

[32] J. R. Siekierzycka, C. Hippius, F. Würthner, R. M. Williams, A. M. Brouwer, J. Am. Chem. Soc. 2010, 132, 1240-1242.

[33] J. R. Siekierzycka, C. Hippius, F. Würthner, R. M. Williams, A. M. Brouwer, Cent. Eur. J. Chem. 2014, 12, 937-952.

[34] Y. Mai, A. Eisenberg, Chem. Soc. Rev. 2012, 41, 59695985.

[35] J. Zhu, R. C. Hayward, J. Am. Chem. Soc. 2008, 130, 7496-7502.

[36] M. Ovesný, P. Křǐžek, J. Borkovec, Z. Švindrych, G. M. Hagen, Bioinformatics 2014, 30, 2389-2390.

[37] B. Mariano, F. Jonas, D. Marcus, W. Volker, W. H. Stefan, New J. Phys. 2006, 8, 275.

[38] T. Dertinger, R. Colyer, G. Iyer, S. Weiss, J. Enderlein, Proc. Natl. Acad. Sci. U. S. A. 2009, 106, 22287-22292. 
Entry for the Table of Contents (Please choose one layout)

Layout 1:

\section{COMMUNICATION}

Enlightened soft matter: A diarylethene photoswitch with a dark open and a fluorescent closed form is introduced as novel and robust label for superresolved photoactivation localization microscopy (PALM) of apolar soft matter structures. Its applicability is rationalized on the basis of a suitable photochemical switching kinetics.

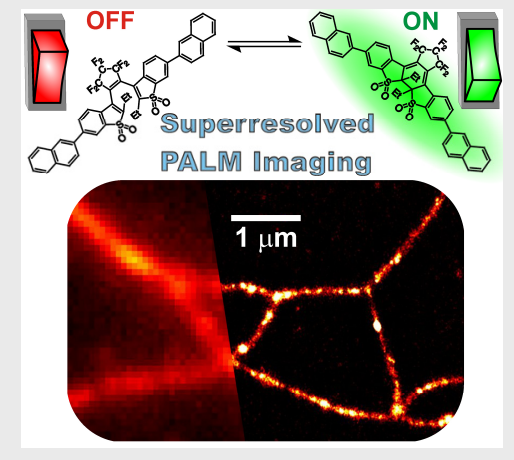

Oleksii Nevskyi, Dmytro Sysoiev, Alex Oppermann Thomas Huhn, and Dominik Wöll*

Page No. - Page No.

Nanoscopic visualization of soft matter using fluorescent diarylethene photoswitches

Layout 2:

\section{COMMUNICATION}

((Insert TOC Graphic here))

Text for Table of Contents
Author(s), Corresponding Author(s)*

Page No. - Page No.

Title 